International Journal of Pure and Applied Mathematics

Volume 92 No. 1 2014, 51-59

ISSN: 1311-8080 (printed version); ISSN: 1314-3395 (on-line version)

url: http://www.ijpam.eu

doi: http://dx.doi.org/10.12732/ijpam.v92i1.4

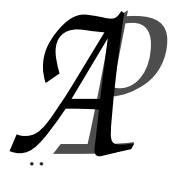

ijpam.eu

\title{
ON CERTAIN RELATIONS FOR $C$-CLOSURE OPERATIONS ON AN ORDERED SEMIGROUP
}

\author{
Thawhat Changphas \\ Department of Mathematics \\ Faculty of Science \\ Khon Kaen University \\ Khon Kaen, 40002, THAILAND
}

\begin{abstract}
In this paper, a relation for $C$-closure operations on an ordered semigroup is introduced, using this relation regular and simple ordered semigroups are characterized.

\section{AMS Subject Classification: $06 \mathrm{~F} 05$}

Key Words: semigroup, ordered semigroup, ideal, regular ordered semigroup, simple ordered semigroup, $C$-closure operation
\end{abstract}

\section{Preliminaries}

It is known that a semigroup $S$ is regular if and only if it satisfies:

$$
A \cap B=A B
$$

for all right ideals $A$ and for all left ideals $B$ of $S$. Using this property, Ponděliček [2] introduced a relation for $C$-closure operations on $S$, and studied some types of semigroups using the relation. The purpose of this paper is to extend Pondělíček's results to ordered semigroups. In fact, we define a relation for $C$-closure operations on an ordered semigroup, and characterize regular and simple ordered semigroups using the relation. Firstly, let us recall some certain definitions and results which are in [2].

Received: November 7, 2013

(c) 2014 Academic Publications, Ltd. url: www.acadpubl.eu 
Let $S$ be a nonempty set. A mapping $\mathbf{U}: \mathrm{Su}(S) \rightarrow \mathrm{Su}(S)$ (The $\operatorname{symbol} \mathrm{Su}(S)$ stands for the set of all subsets of $S$ ) is called a $C$-closure operation on $S$ if, for any $A, B$ in $\operatorname{Su}(S)$, it satisfies:

(i) $\mathbf{U}(\emptyset)=\emptyset$;

(ii) $A \subseteq B \Rightarrow \mathbf{U}(A) \subseteq \mathbf{U}(B)$;

(iii) $A \subseteq \mathbf{U}(A)$;

(iv) $\mathbf{U}(\mathbf{U}(A))=\mathbf{U}(A)$.

For an element $x$ in $S$ we write $\mathbf{U}(x)$ instead of $\mathbf{U}(\{x\})$. A subset $A$ of $S$ is said to be $\mathbf{U}$-closed if $\mathbf{U}(A)=A$; and $A$ is said to be $\mathbf{U}$-open if $S \backslash A$ is $\mathbf{U}$-closed. Let $\mathcal{F}(\mathbf{U})$ denote the set of all $\mathbf{U}$-closed subsets of $S$, and let $\mathcal{O}(\mathbf{U})$ denote the set of all U-open subsets of $S$.

Define a relation $\leq$ on $\mathcal{C}(S)$, the set of all $C$-closure operations on a nonempty set $S$, by

$$
\mathbf{U} \leq \mathbf{V} \text { if and only if } \mathbf{U}(A) \subseteq \mathbf{V}(A) \text { for any } A \text { in } \operatorname{Su}(S) .
$$

A $C$-closure operation $\mathbf{I}$ on $S$ is defined by $\mathbf{I}(\emptyset)=\emptyset$, and $\mathbf{I}(A)=S$ for any nonempty subset $A$ of $S$. A $C$-closure operation $\mathbf{O}$ on $S$ is defined by $\mathbf{O}(A)=A$ for all subsets $A$ of $S$. For any $\mathbf{U}$ and $\mathbf{V}$ in $\mathcal{C}(S)$ it is known that:

(1) $\mathbf{O} \leq \mathbf{U} \leq \mathbf{I}$

(2) $\mathbf{U} \leq \mathbf{V}$ if and only if $\mathcal{F}(\mathbf{V}) \subseteq \mathcal{F}(\mathbf{U})$.

(3) $\mathbf{U} \vee \mathbf{V}, \mathbf{U} \wedge \mathbf{V}$ exist, and

(3.1) $\mathcal{F}(\mathbf{U} \vee \mathbf{V})=\mathcal{F}(\mathbf{U}) \cap \mathcal{F}(\mathbf{V})$,

(3.2) $\mathcal{F}(\mathbf{U} \wedge \mathbf{V})=\{A \cap B \mid A \in \mathcal{F}(\mathbf{U}), B \in \mathcal{F}(\mathbf{V})\}$.

A $C$-closure operation $\mathbf{U}$ on a nonempty set $S$ is said to be a $D$-closure operation if, for any indexed family $\left\{A_{\mathrm{i}} \mid i \in I\right\}$ of subsets of $S$, it satisfies:

$$
\mathbf{U}\left(\bigcup A_{\mathbf{i} \in \mathbf{l}}\right)=\bigcup_{\mathbf{i} \in \mathbf{l}} \mathbf{U}\left(A_{\mathbf{i}}\right) \text {. }
$$

Note that if $\mathbf{U}$ and $\mathbf{V}$ are $D$-closure operations on $S$, then $\mathbf{U} \vee \mathbf{V}$ is a $D$-closure operation on $S$.

For each $C$-closure operation $\mathbf{U}$ on a nonempty set $S$, a $D$-closure operation $\mathbf{U}^{*}$ is defined on $S$ by

$$
\mathbf{U}^{*}(A)=\{x \mid \mathbf{U}(x) \cap A \neq \emptyset\}
$$


for any $A$ in $\operatorname{Su}(S)$.

It is known that:

(1) $\mathbf{I}^{*}=\mathbf{I}, \mathbf{O}^{*}=\mathbf{O}$.

(2) For any $\mathbf{U}, \mathbf{V}$ in $\mathcal{C}(S), \mathbf{U} \leq \mathbf{V}$ implies $\mathbf{U}^{*} \leq \mathbf{V}^{*}$.

(3) For any $\mathbf{U}$ in $\mathcal{C}(S)$, the following conditions are equivalent:

(3.1) $\mathbf{U}$ is a $D$-closure operation;

(3.2) $\mathbf{U}=\mathbf{U}^{* *}$;

(3.3) $\mathcal{F}(\mathbf{U})=\mathcal{O}\left(\mathbf{U}^{*}\right)$;

(3.4) $\mathcal{O}(\mathbf{U})=\mathcal{F}\left(\mathbf{U}^{*}\right)$.

(4) For a $D$-closure operation $\mathbf{U}$ on $S, \mathbf{U} \leq \mathbf{U}^{*}$ or $\mathbf{U}^{*} \leq \mathbf{U}$ implies $\mathbf{U}=\mathbf{U}^{*}$.

\section{Main Results}

An ordered semigroup [1] is defined to be a semigroup $(S, \cdot)$ together with a partial order $\leq$ that is compatible with the semigroup operation, meaning that for $x, y, z \in S$,

$$
x \leq y \Rightarrow z x \leq z y, x z \leq y z .
$$

Let $(S, \cdot, \leq)$ be an ordered semigroup. If $A, B$ are nonempty subsets of $S$, we write $A B$ for the set of all elements $x y$ in $S$ such that $x$ in $A$ and $y$ in $B$, and write

$$
(A]=\{x \in S \mid x \leq a \text { for some } a \in A\} .
$$

For an element $x$ in $S$, we write $A x$ and $x A$ for $A\{x\}$ and $\{x\} A$, respectively. In [4], the following conditions hold:

(1) $A \subseteq(A]$;

(2) $A \subseteq B \Rightarrow(A] \subseteq(B]$;

(3) $(A](B] \subseteq(A B]$;

(4) $((A](B]]=(A B]$;

(5) $(A \cup B]=(A] \cup(B]$. 
The following concepts can be found in [3]. Let $(S, \cdot, \leq)$ be an ordered semigroup. A nonempty subset $A$ of $S$ is called a left (respectively, right) ideal [3] of $S$ if it satisfies:

(i) $S A \subseteq A$ (respectively, $A S \subseteq A$ );

(ii) $A=(A]$, that is, for $x \in A$ and $y \in S, y \leq x$ implies $y \in A$.

If $A$ is both a left and a right ideal of $S$, then $A$ is called an ideal of $S$.

Let $(S, \cdot, \leq)$ be an ordered semigroup. If $A$ is a nonempty subset of $S$, then $(A \cup S A]$ (respectively, $(A \cup A S],(A \cup S A \cup A S \cup S A S])$ are left (respectively, right, two-sided) ideals of $S$.

Definition 1. Let $(S, \cdot, \leq)$ be an ordered semigroup. Define a relation $\varrho$ on $\mathcal{C}(S)$ by

$$
\mathbf{U} \varrho \mathbf{V} \text { if and only if } A \cap B=(A B]
$$

for all nonempty subsets $A$ in $\mathcal{F}(\mathbf{U})$ and for all nonempty subsets $B$ in $\mathcal{F}(\mathbf{V})$.

Lemma 2. Let $(S, \cdot \leq)$ be an ordered semigroup, and let $\mathbf{U}, \mathbf{U}^{\prime}, \mathbf{V}, \mathbf{V}^{\prime}$ be $C$-closure operations on $S$ such that $\mathbf{U} \varrho \mathbf{V}$. If $\mathbf{U} \leq \mathbf{U}^{\prime}$ and $\mathbf{V} \leq \mathbf{V}^{\prime}$, then $\mathbf{U}^{\prime} \varrho \mathbf{V}^{\prime}$.

Proof. This follows directly from the definition of $\varrho$.

Let $(S, \cdot, \leq)$ be an ordered semigroup. Define a mapping $\mathbf{L}$ on $\mathrm{Su}(S)$ by $\mathbf{L}(\emptyset)=\emptyset$, and

$$
\mathbf{L}(A)=(A \cup S A]
$$

for any nonempty subset $A$ of $S$. It is easy to verify that $\mathbf{L}$ is a $C$-closure operation on $S$. Note that $\mathcal{F}(\mathbf{L})$ is the set of all left ideals of $S$ (including empty set). Indeed, if $L$ is a left ideal of $S$, then

$$
L \subseteq \mathbf{L}(L)=(L \cup S L] \subseteq(L]=L
$$

hence $L \in \mathcal{F}(\mathbf{L})$. Conversely, if $L \in \mathcal{F}(\mathbf{L})$, then $L=\mathbf{L}(L)=(L \cup S L]$, and thus $L$ is a left ideal of $S$. Similarly, we define a $C$-closure operation on $S$ by $\mathbf{R}(\emptyset)=\emptyset$, and

$$
\mathbf{R}(A)=(A \cup A S]
$$

for any nonempty subset $A$ of $S . \mathcal{F}(\mathbf{R})$ is the set of all right ideals of $S$ (including empty set). 
Lemma 3. Let $(S, \cdot, \leq)$ be an ordered semigroup. Then $\mathbf{L}$ and $\mathbf{R}$ are $D$-closure operations on $S$.

Proof. Let $\left\{A_{\mathrm{i}} \mid i \in I\right\}$ be an indexed family of subsets of $S$. We have

$$
\begin{aligned}
& \mathbf{L}\left(\bigcup_{\mathrm{i} \in \mathrm{l}} A_{\mathrm{i}}\right)=\left(\left(\bigcup_{\mathrm{i} \in \mathrm{l}} A_{\mathrm{i}}\right) \bigcup S\left(\bigcup_{\mathrm{i} \in \mathrm{l}} A_{\mathrm{i}}\right)\right] \\
& =\left(\left(\bigcup_{\mathrm{i} \in \mathrm{I}} A_{\mathrm{i}}\right) \bigcup\left(\bigcup_{\mathrm{i} \in \mathrm{I}}\left(S A_{\mathrm{i}}\right)\right)\right] \\
& =\left(\bigcup_{\mathrm{i} \in \mathrm{I}}\left(A_{\mathrm{i}} \cup S A_{\mathrm{i}}\right)\right] \\
& =\bigcup_{\mathrm{i} \in \mathrm{l}}\left(A_{\mathrm{i}} \cup S A_{\mathrm{i}}\right] \\
& =\bigcup_{\mathbf{i} \in \mathbf{I}} \mathbf{L}\left(A_{\mathbf{i}}\right)
\end{aligned}
$$

Then $\mathbf{L}$ is a $D$-closure operation on $S$. Similarly, $\mathbf{R}$ is a $D$-closure operation on $S$.

Theorem 4. Let $(S, \cdot, \leq)$ be an ordered semigroup, and let $\mathbf{U}, \mathbf{V}$ be $C$-closure operations on $S$. Then $\mathbf{U} \varrho \mathbf{V}$ if and only if $\mathbf{R} \leq \mathbf{U}, \mathbf{L} \leq \mathbf{V}$ and $x \in(\mathbf{U}(x) \mathbf{V}(x)]$ for all $x$ in $S$.

Proof. Assume $\mathbf{U} \varrho \mathbf{V}$. Clearly, $S \in \mathcal{F}(\mathbf{V})$. If $A \in \mathcal{F}(\mathbf{U})$, then $A=A \cap S=$ $(A S]$; hence

$$
\mathbf{R}(A)=(A \cup A S]=((A S] \cup A S]=((A S]]=(A S]=A .
$$

This shows that $A \in \mathcal{F}(\mathbf{R})$; thus $\mathbf{R} \leq \mathbf{U}$. Similarly, $\mathbf{L} \leq \mathbf{V}$. Let $x$ be an element of $S$. Since $\mathbf{U}(x) \in \mathcal{F}(\mathbf{U})$ and $\mathbf{V}(x) \in \mathcal{F}(\mathbf{V})$, we have $x \in \mathbf{U}(x) \cap \mathbf{V}(x) \subseteq$ $(\mathbf{U}(x) \mathbf{V}(x)]$ as required.

Conversely, assume that $\mathbf{R} \leq \mathbf{U}, \mathbf{L} \leq \mathbf{V}$ and $x \in(\mathbf{U}(x) \mathbf{V}(x)$ ] for all $x$ in $S$. To show that $\mathbf{U} \varrho \mathbf{V}$, let $A \in \mathcal{F}(\mathbf{U})$ and $B \in \mathcal{F}(\mathbf{V})$ be nonempty. Then $A \in \mathcal{F}(\mathbf{R})$ and $B \in \mathcal{F}(\mathbf{L})$. We have

$$
(A B] \subseteq(A S] \subseteq(A]=A \text { and }(A B] \subseteq(S B] \subseteq(B]=B .
$$

This shows that $(A B] \subseteq A \cap B$. For the reverse inclusion, let $x$ be an element of $A \cap B$. Then $\mathbf{U}(x) \subseteq \mathbf{U}(A)=A$. Similarly, $\mathbf{V}(x) \subseteq B$. By

$$
x \in \mathbf{U}(x) \mathbf{V}(x) \subseteq A B \subseteq(A B]
$$


follows $A \cap B \subseteq(A B]$.

An ordered semigroup $(S, \cdot, \leq)$ is said to be left regular if $x \in\left(S x^{2}\right]$ for every $x$ in $S$, or equivalently, $x \in\left(x^{2} \cup S x^{2}\right]$ for every $x$ in $S$. A right regular ordered semigroup is defined dually. $S$ is said to be regular if $x \in(x S x]$ for every $x$ in $S$, or equivalently, $x \in\left(x^{2} \cup x S x\right]$ for every $x$ in $S$. These concepts can be found in [4].

Theorem 5. An ordered semigroup $(S, \cdot, \leq)$ is regular if and only if $\mathbf{R} \varrho \mathbf{L}$.

Proof. Assume that $S$ is regular. Then for any $x$ in $S$ we have

$$
x \in(x S x]=((x](S x]] \subseteq(\mathbf{R}(x) \mathbf{L}(x)] ;
$$

hence $\mathbf{R} \varrho \mathbf{L}$ by Theorem 4 .

Conversely, $\mathbf{R} \varrho \mathbf{L}$ implies $S$ is regular since, for any $x$ in $S$, we have

$$
\begin{aligned}
x \in(\mathbf{R}(x) \mathbf{L}(x)] & =((x \cup x S](x \cup S x]] \\
& =((x \cup x S)(x \cup S x)] \\
& \subseteq\left(x^{2} \cup x S x\right] .
\end{aligned}
$$

Let $(S, \cdot, \leq)$ be an ordered semigroup. We denote the $D$-closure operation $\mathbf{R} \vee \mathbf{L}$ on $S$ by $\mathbf{M}$. Note that $\mathcal{F}(\mathbf{M})$ is the set of all ideals of $S$ (including empty set).

Theorem 6. The following statements are equivalent on an ordered semigroup $(S, \cdot, \leq)$ :

(1) $\mathbf{L} \varrho \mathbf{L}$;

(2) $\mathbf{L} \varrho \mathbf{M}$;

(3) $S$ is left regular and $\mathbf{R} \leq \mathbf{L}$.

Proof. $(1) \Rightarrow(2)$. Since $\mathbf{L} \leq \mathbf{M}$, it follows by Lemma 2 that $\mathbf{L} \varrho \mathbf{M}$.

$(2) \Rightarrow(3)$. Assume $\mathbf{L} \varrho \mathbf{M}$. Then $\mathbf{R} \leq \mathbf{L}$ by Theorem 4; hence $\mathbf{M}=\mathbf{L}$. For any $x$ in $S$, we have

$$
\begin{aligned}
x \in(\mathbf{L}(x) \mathbf{M}(x)] & =(\mathbf{L}(x) \mathbf{L}(x)] \\
& =((x \cup S x](x \cup S x]] \\
& =((x \cup S x)(x \cup S x)] \\
& \subseteq\left(x^{2} \cup x S x \cup S x^{2} \cup S x S x\right]
\end{aligned}
$$




$$
\begin{aligned}
& =((x \cup x S) x \cup S(x \cup x S) x] \\
& \subseteq(\mathbf{R}(x) x \cup S \mathbf{R}(x) x] \\
& \subseteq(\mathbf{L}(x) x \cup S \mathbf{L}(x) x] \\
& \subseteq\left(x^{2} \cup S x^{2}\right] .
\end{aligned}
$$

This shows that $S$ is left regular.

$(3) \Rightarrow(1)$. Assume that $S$ is left regular and $\mathbf{R} \leq \mathbf{L}$. Then, for any $x$ in $S$, we have

$$
x \in\left(S x^{2}\right]=((S x](x]] \subseteq(\mathbf{L}(x) \mathbf{L}(x)] .
$$

By Theorem 4, $\mathbf{L} \varrho \mathbf{L}$.

Theorem 7. The following statements are equivalent on an ordered semi$\operatorname{group}(S, \cdot, \leq)$ :

(1) $\mathbf{R} \varrho \mathbf{R}$;

(2) $\mathbf{M} \varrho \mathbf{R}$;

(3) $S$ is right regular and $\mathbf{L} \leq \mathbf{R}$.

Proof. The proof is left-right dual of Theorem 6.

An ordered semigroup $(S, \cdot, \leq)$ is said to be left simple (right simple) if it contains no proper left (right) ideal, and $S$ is said to be simple if it contains no proper ideal.

Lemma 8. Let $(S, \cdot, \leq)$ be an ordered semigroup. Then:

(1) $S$ is left simple if and only if $\mathbf{L}=\mathbf{I}$;

(2) $S$ is right simple if and only if $\mathbf{R}=\mathbf{I}$;

(3) $S$ is simple if and only if $\mathbf{M}=\mathbf{I}$.

Proof. Assume that $S$ is a left simple. If $A$ is a nonempty subset of $S$, then

$$
\mathbf{L}(A)=(A \cup S A]=S=\mathbf{I}(A) ;
$$

hence $\mathbf{L}=\mathbf{I}$. Conversely, if $A$ is a left ideal of $S$, then

$$
S=\mathbf{I}(A)=\mathbf{L}(A)=(A \cup S A] \subseteq A \subseteq S,
$$

i.e., $A=S$. This proves (1). The statements (2) and (3) are proved similarly. 
Lemma 9. $\mathbf{L} \vee \mathbf{R}^{*}=\mathbf{I}=\mathbf{L}^{*} \vee \mathbf{R}$.

Proof. Let $A$ be an element of $\mathcal{F}\left(\mathbf{L} \vee \mathbf{R}^{*}\right)$; then $A \in \mathcal{F}(\mathbf{L})$ and $S \backslash A \in \mathcal{F}(\mathbf{R})$. If $A$ is a proper subset of $S$, then $(S \backslash A) A \subseteq A \cap(S \backslash A)$. This is a contradiction. Thus $A=S$, and hence $\mathbf{L} \vee \mathbf{R}^{*}=\mathbf{I}$. Similarly, $\mathbf{L}^{*} \vee \mathbf{R}=\mathbf{I}$.

Theorem 10. The following statements are equivalent on an ordered $\operatorname{semigroup}(S, \cdot, \leq)$ :

(1) $S$ is simple;

(2) $\mathbf{L} \leq \mathbf{M}^{*}$

(3) $\mathbf{R} \leq \mathbf{M}^{*}$;

(4) $\mathbf{M}^{*} \varrho \mathbf{I}$

(5) $\mathbf{I} \varrho \mathbf{M}^{*}$.

Proof. If $S$ is simple, then $\mathbf{M}=\mathbf{I}$; hence

$$
\mathbf{L} \leq \mathbf{I}=\mathbf{I}^{*}=\mathbf{M}^{*} .
$$

Conversely, assume that $\mathbf{L} \leq \mathbf{M}^{*}$. Since $\mathbf{R} \leq \mathbf{M}$, we have $\mathbf{R}^{*} \leq \mathbf{M}^{*}$. By Lemma 9,

$$
\mathbf{I}=\mathbf{L} \vee \mathbf{R}^{*} \leq \mathbf{M}^{*}
$$

Thus $\mathbf{M}^{*}=\mathbf{I}, \mathbf{M}=\mathbf{I}$. This proves $(1) \Leftrightarrow(2)$. For $(1) \Leftrightarrow(3)$ can be proved similarly. By Theorem $4,(5) \Rightarrow(1)$ and $(4) \Rightarrow(1)$. If $S$ is simple, then $\mathbf{M}=\mathbf{I}=\mathbf{I}^{*}$. Since $\left(S^{2}\right]=S$, it follows that $\mathbf{I} \varrho \mathbf{I}$. Thus (4) and (5) hold.

Lemma 11. An ordered semigroup $(S, \cdot, \leq)$ is left simple if and only if $\mathbf{R} \leq \mathbf{L}^{*}$.

Proof. If $S$ is left simple, then $\mathbf{L}=\mathbf{I}$; hence

$$
\mathbf{R} \leq \mathbf{I}=\mathbf{I}^{*}=\mathbf{L}^{*}
$$

Conversely, assume that $\mathbf{R} \leq \mathbf{L}^{*}$. By Lemma 9,

$$
\mathbf{I}=\mathbf{L}^{*} \vee \mathbf{R} \leq \mathbf{L}^{*} \leq \mathbf{I}
$$

Hence $\mathbf{L}^{*}=\mathbf{I}$, and so $\mathbf{L}=\mathbf{I}$.

Theorem 12. The following are equivalent for an ordered semigroup $(S, \cdot, \leq)$ : 
(1) $S$ is left simple;

(2) $\mathbf{L}^{*} \varrho \mathbf{I}$

(3) $\mathbf{L} \varrho \mathbf{L}^{*}$.

Proof. This can be proved as Theorem 10.

Lemma 13. An ordered semigroup $(S, \cdot, \leq)$ is right simple if and only if $\mathbf{L} \leq \mathbf{R}^{*}$.

Proof. This can be proved as Lemma 11.

Theorem 14. The following are equivalent for an ordered semigroup $(S, \cdot, \leq)$ :

(1) $S$ is right simple;

(2) $\mathbf{I} \varrho \mathbf{R}^{*}$;

(3) $\mathbf{R}^{*} \varrho \mathbf{R}$.

Proof. This can be proved as Theorem 10.

\section{References}

[1] G. Birkhoff, Lattice Theory, 25, Rhode Island, American Mathematical Society Colloquium Pub., Am. Math. Soc., Providence, 1984.

[2] B. Ponděliček, On a certain relation for closure operations on a semigroup, Czechkoslovak Mathematical Journal, 20 (1970), no. 2, 220-231.

[3] N. Kehayopulu, On weakly prime ideals of ordered semigroups, Mathematica Japonica, 35 (1990), no. 6, 1051-1056.

[4] N. Kehayopulu, M. Tsingelis, On left regular ordered semigroups, Southeast Asian Bulletin of Mathematics, 25 (2002), 609-615. 
